\title{
Ventajas y usos de Twitter, como herramienta de mejora de la educación universitaria
}

Fernando J. Garrigos-Simón ${ }^{\mathbf{a}}$, Juan Vte. Oltra Gutiérrez ${ }^{\mathrm{b}}$, Yeamduan Narangajavana $^{c}$ y Sofia Estellés Miguel ${ }^{d}$

anniversitat Politècnica de València, fgarrigos@doe.upv.es, ${ }^{\text {b, d, }}$ Universitat Politècnica de València ${ }^{c}$ Universitat Jaume I

\begin{abstract}
This communication aims to analyze the possibilities of the social media Twitter, as an important tool for improving teaching methods in high level education. Specifically, the article studies several ways and possibilities of this social media, in order to provide different applications and services for students, lecturers and organizations.

The paper concentrates in the study of the advantages and uses of Twitter, as a previous step to implement the tool as a relevant application to be used in the universities, although it also mention some disadvantages and methodological applications. The paper is the starting point of a project developed at the Escuela Técnica Superior de Ingeniería Informática de la Universidad Politécnica de Valencia (hereinafter ETSINF-UPV), and financed with the program PIME 2015-2016
\end{abstract}

Keywords: Twitter, Social Media, education, learning

\begin{abstract}
Resumen
Esta comunicación pretende analizar las pobililidades de la red social Twitter, como herramienta importante para mejorar los metodos de ensañanza en la educación superior. Específicamente, el artículo estudia diversos modos y posibilidades de esta red social, con el objeto de proveer diferentes aplicaciones y servicios para estudiantes, profesores $y$ organizaciones.

El trabajo se concentra en el estudio de las ventajas y usos de Twitter, como paso previo a implementar esta herramienta como aplicación relevante para su utilización en las universidades, aunque señala también posibles desventajas y aplicaciones metodológicas. El artículo es el punto de partida de un proyecto más amplio desarrollado en la Escuela Técnica Superior de Ingeniería Informática de la Universidad Politécnica de València (ETSINFUPV), y financiado con el programa PIME 2015-2016
\end{abstract}

Palabras clave: Twitter, redes sociales, educación, aprendizaje. 
Acknowledgements: We are grateful for financial help from the Universidad Politécnica de Valencia, within the program. PIME 2015-16

\section{Introducción}

El entorno educativo actual demanda la aplicación de innovaciones importantes en la cultura educativa, y obviamente en los modos de enseñar y aprender (Garrigós et al., 2015).

Concretamente es esencial el uso de estrategias educativas centradas en metodologías activas, que promuevan la participación de los alumnos, y el uso de procesos de coaprendizaje enfocados, como aspecto central, en las características de los nuevos estudiantes.

En un entorno caracterizado por la evolución de la Web 2.0, su transformación y desarrollo con la web semántica o Web 3.0 (Garrigós et al., 2012), la emergencia de la sociedad ubicua, "un mundo donde la informática está presente en todos lados simultáneamente" (Westerlund and Kaivo-oja, 2012:143), y específicamente la conformación de entornos de aprendizaje ubicuos, que pueden integrar la colaboración de la enseñanza, el contenido de la enseñanza y los servicios de la enseñanza (Yang, 2006), la educación demanda y requiriere un profundo cambio en los métodos tradicionales de enseñanza y aprendizaje. En este marco, la utilización de las redes sociales como mecanismos de aprendizaje y creación de conocimiento, o como fuentes esencial es para explorar y descubrir las continuas innovaciones y tendencias en el entorno, y las contribuciones de nuevos expertos.

Dado que estos análisis son extensos, por la variedad y amplitud de posibles metodologías, y dada la existencia de múltiples redes sociales con capacidades didácticas, en este artículo nos planteamos el análisis concreto de una de ellas, Twitter, por su importancia en la sociedad, y por su posible relevancia práctica en el contexto educativo. En este sentido, este artículo planteará esencialmente el análisis de las ventajas y usos de Twitter como una herramienta importante para mejorar la educación y el aprendizaje en la educación universitaria, observando algunas desventajas de su uso, y diversas ideas metodológicas de aplicación en el aula y en los planes educativos.

\section{Twitter}

Twitter es un servicio de micro blogs, creado en California en marzo de 2006 por Jack Dorsey, Noah Glass, Evan Williams y Biz Stone. La plataforma pronto se expandió en todo el mundo, publicándose en múltiples lenguas. Por ejemplo, en 2009 apareció la versión en Español (Castellano y Oltra, 2015).

Descrita como "el SMS de Internet", Twitter se ha convertido en uno de las 10 plataformas web más visitadas en Internet (Maduewesi, 2013). Hoy en día, tiene más de 320 millones de usuarios actives, es decir, usuarios que al menos lanzan un "tweet" al mes, y da soporte en más de 35 lenguas (Twitter, 2016), diversos alfabetos, y a la vez permite traducción 
automática. Específicamente, al menos 1,4 millones de los usuarios activos son españoles (Sanmarco, 2015). Además, Twitter está accesible a través de múltiples plataformas (web, teléfonos inteligentes, tabletas...).

La característica peculiar y principal de esta red es la de ofrecer a los usuarios enviar mensajes cortos, conocidos como "tweets", con un máximo de 140 caracteres. Los usuarios pueden suscribirse a los tweets de otros usuarios, pudiéndoles seguirles como "followers". Aunque por defecto, los mensajes son públicos, ellos pueden a su vez ser difundidos privadamente a ciertos seguidores.

A su vez, y opuestamente a otras redes sociales, la relación establecida entre los usuarios no es uno a uno, sino que opuestamente, las comunicaciones son múltiples y de forma simultánea uno-a-muchos. Este hecho, que parece que la coloca fuera de las redes sociales, es uno de los aspectos que nos hace considerar su apropiabilidad para enseñar, dado que podemos virtualizar la conexión "uno-a-muchos" que el profesor establece con sus estudiantes en el aula.

\section{Ventajas y usos de Twitter para la educación universitaria.}

El uso de Twitter para la educación tiene muchas ventajas. Entre ellas podemos mencionar su facilidad para: utilizar listas o "hastags" (los usuarios etiquetan las publicaciones o eventos), con el fin de agrupar por grupos, temas... permitiendo la posibilidad de propagar las opiniones de las personas fácilmente; buscar, mediante palabras o "hastags", los tweets relacionados, lo que permite localizar personas o información interesante, rápida e instantáneamente, relacionada con la materia a desarrollar; ser viral, dado que los contribuidores pueden "retuitear" un "tweet" de otros usuarios, o difundir fotos, noticias...; ser un acceso multidispositivo (ej. los teléfonos inteligentes siembre están en el bolsillo de los estudiantes); acceder a los denominados "Trending Topics", o temas más relevantes o hablados en cada momento, que pueden ser filtrados por localización. Además entre las ventajas de Twitter para la enseñanza y el aprendizaje, podemos subrayar:

1. Es accesible, ya que hoy en día está disponible desde casi cualquier plataforma y dispositivo que permita la conexión a Internet (García, 2015) 2. La inmediatez (Dunlap y Lowenthal, 2009; Tess, 2013). En este sentido, es dinámico, ya que la información se presenta en tiempo real (García, 2015), un hecho que facilita el acceso a la información dentro de una institución (Noguera, 2015). 3. Aumenta el aprendizaje en general (Thoms y Eryilmaz, 2015), promueve la educación autónoma, auto dirigida e independiente para los estudiantes (Noguera, 2015) y sobre todo ayuda al aprendizaje informal y autónomo de los estudiantes, que pueden descubrir recursos y herramientas de se pueden aplicar de manera efectiva en sus cursos (Dunlap y Lowenthal, 2009; Grosseck y Holotescu, 2008; Tess, 2013) 4. Produce una lectura ágil, derivada de su brevedad (140 caracteres) (García, 2015). 5. El hecho de tener que expresar en sólo 140 caracteres ideas u opiniones sobre un tema, ayuda a los estudiantes en sus habilidades de escritura. Por lo tanto, focaliza la atención de los estudiantes, y la búsqueda de objetivos relacionados con el plan de estudio, pero no atados o restringidos al mismo (Grosseck y Holotescu, 2008), y se promueve el aprender a

(cc)) BY-NC-ND 2016, Universitat Politècnica de València

Congreso In-Red (2016) 
escribir para un público en particular (Dunlap y Lowenthal, 2009). 6. Facilita la búsqueda, intercambio y síntesis de la información relevante de forma rápida (Miguel y Fernández, 2013). Por ejemplo, los artículos son transformados por la red social, que resume, y los hace atractivos para los usuarios. Este hecho facilita la búsqueda de la información más relevante.

7. Es interactivo (Dunlap y Lowenthal, 2009; Tess, 2013, Thoms y Eryilmaz, 2015), permitiendo ver otras publicaciones, intervenir, compartir o guardar mensajes cuando se marcan como favoritos (García-Suárez et al, 2015). 8. Facilita la interacción y el trabajo colaborativo entre los estudiantes y profesores, entre los estudiantes (García-Suárez et al, 2015; Grosseck y Holotescu, 2008) (abriendo la puerta a los métodos de enseñanza centrados en el estudiante, y a la redefinición de los roles de profesores y estudiantes (Noguera, 2015)), entre los profesores (Carpenter y Krutka, 2014), la colaboración entre las diversas escuelas y países (Grosseck y Holotescu, 2008), o incluso la comunicación directa con los padres, que pueden también seguir las actividades de sus hijos. Por ejemplo, según Carpenter y Krutka (2014), Twitter puede permitir que los educadores aprendan los unos de los otros de forma aparentemente significativa, mientras que Grosseck y Holotescu (2008) afirma que el hecho de participar en la educación y el intercambio de las mejores prácticas utilizadas por los profesores, cambia la dinámica en el aula.

9. Promueve la motivación de los estudiantes, ya que proporciona elementos de disfrute y la presencia social (Noguera, 2015), y mejorar el ambiente de trabajo (Miguel y Fernández, 2013). Además, permite la conexión con estudiantes de otras escuelas y países. 10. Promueve la colaboración y el trabajo en red, así como la innovación y la creatividad (Noguera, 2015). Facilita seguir a las personas que contribuyen en los asuntos que se están estudiando; el acceso a los profesionales, con o sin retroalimentación (Dunlap y Lowenthal, 2009; Guzmán et al, 2012); la difusión de publicaciones y material didáctico (Grosseck y Holotescu, 2008); la tarea de estudiantes como pequeños emisores de información (Moody, 2010), ya que pueden compartir las cosas que pueden encontrar (Dunlap y Lowenthal, 2009), por ejemplo utilizando Twitter como una revista del club para compartir documentos o informaciones relacionadas (Barreto y Jiménez , 2010); a su vez los estudiantes pueden utilizar los tweets con el fin de enviar preguntas y observaciones al grupo durante las actividades en el aula, o, para los asistentes a una conferencia, Twitter puede proporcionar una forma sencilla de compartir, con otros en el evento, pensamientos acerca de las sesiones y actividades específicas, y con los que no pueden asistir, la cobertura en vivo de estos eventos (Großeck y Holotescu de 2008), ayudando a la tormenta de ideas (Barreto y Jiménez, 2010) o a difundir las actualizaciones de la conferencia a los no asistentes (Anikeeva, y Bywood, 2013)

11. En concreto, Twitter facilita la conectividad y el intercambio de recursos, lo que facilita el aprendizaje colaborativo (Carpenter, 2014), y puede tener un impacto positivo en la creación de un ambiente de trabajo colaborativo (Rinaldo et la., 2011). Ayuda a mejorar el intercambio de material de recursos, la búsqueda y asignación de tareas, anuncios, o la negociación de la reprogramación de la clase. Además, igual que Facebook, los impactos negativos de su posible uso para el entretenimiento por parte de los alumnos es relativamente bajo en comparación con los beneficios positivos (Falahah y Rosmala 2012).

(cc)) EY-NC-ND 2016, Universitat Politècnica de València 
12. Tiene una alta funcionalidad, ya que permite incrustar imágenes, vídeos y enlaces (García, 2015), y el uso integrado de herramientas y presentaciones de vídeo (Moody, 2010). Además, ofrece la posibilidad de conferencias virtuales a través de "streaming" (aunque esto es una funcionalidad externa), con las posibilidades de chatear. Este hecho también promueve la tutoría entre iguales (Dunlap y Lowenthal, 2009), ya que permite compartir con los demás imágenes, videos, documentos, presentaciones, etc. 13. La aplicación tiene una posibilidad reciente de encuestas, con posibles encuestas sobre temas relevantes a diversos miembros.

14. Permite una fácil organización del tiempo (Dunlap y Lowenthal, 2009), y la organización a través de "hashtags" y fechas de publicación, (García, 2015). Esto facilita realizar debates a partir de un "hashtag". Además, es útil para la gestión de proyectos (Grosseck y Holotescu, 2008)

15. No es invasivo, la herramienta se abre cuando se quiere. También permite el anonimato (García-Suárez et al., 2015), lo que facilita la inclusión, la equidad, el aprendizaje permanente y la movilidad de los educandos (Noguera, 2015) 16. Mejora el diálogo (Moody, 2010), y promueve la comprensión de los materiales, la comunicación y el fomento del aprendizaje de los estudiantes. (García, 2015), lo que facilita cambios en procesos cognitivos y formas de aprendizaje (Noguera, 2015). 17. Promueve la presencia docente, o la capacidad de los maestros para apoyar y mejorar la presencia social y cognitiva a través de la gestión de la instrucción, la construcción de la comprensión, y la instrucción directa, dado que Twitter ayuda a los profesores a participar en interacciones con los estudiantes (Dunlap y Lowenthal, 2009; Tess, 2013). Además, facilita la centralización de las actividades y fuentes de información y la coordinación del trabajo, lo que permite el monitoreo de los puntos principales y el seguimiento de las actividades (Miguel y Fernández, 2013)

18. Twitter, como Facebook, está reconocido por capacitar a los estudiantes a construir comunidades y aprender al mismo tiempo fuera del aula (Mendoza, 2009; Venkatesh et al, 2014). Twitter es una herramienta valiosa para crear o construir un sentido de comunidad y generar confianza y seguridad (Grosseck y Holotescu, 2008; Moody, 2010; Thoms y Eryilmaz, 2015; Wright, 2010). También es un importante mecanismo para fomentar el intercambio, la conversación y la relación (Falahah y Rosmala 2012). Además, promueve la comunicación directa entre los usuarios, un hecho que facilita el acercamiento entre los usuarios (Miguel y Fernández, 2013)

19. Twitter aumentar el compromiso del estudiante y el sentido de pertenencia (Guzmán et al., 2012), e incluso la empatía con la materia (Sullivan, 2012). Particularmente, Welch y Bonnan-White (2012) afirman que Twitter aumenta la participación de los estudiantes en el aula. Además Evans (2014) encontró una correlación positiva entre la cantidad de uso de Twitter y la participación de los estudiantes en actividades asociadas a la universidad, tales como la organización de su vida social y el intercambio de información. 20. Este beneficio de la participación es especialmente importante también en el caso de los cursos en línea o virtuales, ya que su uso en este caso, permite el intercambio de preguntas, ideas o tareas entre todos los participantes (Dunlap y Lowenthal, 2009), y conduce a una mayor cohesión dentro del grupo y en una reducción del número de alumnos que abandonan el curso (Guzmán et al., 2012).

(cc) EY-NC-ND 2016, Universitat Politècnica de València

Congreso In-Red (2016) 
21. Ayuda a superar los límites espaciales y temporales de la clase (García-Suárez et al., 2015), lo que permite extender el trabajo fuera del aula, a partir de preguntas formuladas, tareas subsiguientes con recordatorios de plazos, e incluso plantear cuestiones de futuras clases o crear reuniones virtuales, contribuyendo por lo tanto a la personalización del aprendizaje, (Noguera, 2015).

22. Los estudiantes mejoran su percepción de su navegación social. Este hecho, junto con la facilidad de uso, la usabilidad y experiencia general, y su mayor confianza y sentido de comunidad, les ayuda a aprender fuera del aula y construir comunidades. 23. Por otra parte, permite mantener una relación constante, por ejemplo después de que termine un curso. Por lo tanto, el uso de Twitter por estudiantes y profesores no viene limitado por la estructura de la lección o el tiempo de un semestre, lo que permite el asesoramiento continuo a los estudiantes académicamente y profesionalmente (Dunlap y Lowenthal, 2009; Moody, 2010). Por otra parte, las comunicaciones de Twitter mejoran la participación y engarzamiento de los estudiantes y profesores en el proceso de aprendizaje en modos que trascienden las actividades tradicionales de clase (Junco et al., 2011) 24. Se trata de una plataforma viable para la "metacognición", o la práctica de pensar y reflexionar sobre el aprendizaje (Grosseck y Holotescu, 2008), a la vez que, cuando se utiliza correctamente, instiga a procesos cognitivos complejos (Venkatsh, 2014) 25. Como es una herramienta popular y novedosa, por lo general es bien recibida entre los estudiantes (García, 2015). No podemos olvidar que se trata de una tecnología de redes sociales muy reconocible, y, como herramienta que reconocen como propia, aumenta la motivación, el rendimiento académico y la participación (Noguera, 2015). 26. Mejora la participación en el aula especialmente de aquellos alumnos más introvertidos, para los cuales es difícil exponer sus puntos de vista en voz alta (Guzmán et al., 2012). Mediante el uso de Twitter, los estudiantes pueden abrirse a sus sentimientos y deficiencias (Junco et al., 2011). Por lo tanto, permite que el establecimiento de la comunicación entre aquellos estudiantes inhibidos a hablar directamente con el profesor (Carpenter, 2014). Además, de acuerdo con Guzmán et al., (2012) ayuda a evitar que las bajadas de atención durante una clase, y aumenta la receptividad de los estudiantes, al fomentar la participación activa de vez en cuando.

27. Se puede utilizar para muchos propósitos en el aula. Por ejemplo Stieger y Burger (2010) analizan y encuentran su uso positivo para evaluar propuestas de calidad de la enseñanza, o para evaluar cursos formativamente.

28. Por otra parte, Twitter puede ser usado por ejemplo en las bibliotecas como servicios de referencia. Por ejemplo, los estudiantes o profesores podrían estar involucrados en una cuenta de Twitter para aprender acerca de eventos o acontecimientos de una biblioteca, libros nuevos, o para obtener respuestas a preguntas como usuarios de la biblioteca (Grosseck y Holotescu, 2008).

\section{Usos y desventajas de Twitter}

El uso de la red puede ser diverso. Por ejemplo, en nuestro proyecto estamos intentando utilizarla en dos sentidos. El primero de ellos es como mecanismo válido de aprendizaje, y de desarrollo de diversos desempeños educativos. En este sentido, y aunque estamos en un 
proceso pionero, estamos estudiando la influencia que tiene su uso, y la relación entre distintas aplicaciones y facilidades de Twitter, en aspectos como las notas y el desempeño de los estudiantes, su motivación, o su percepción de enseñanza de aspectos formales o informales en diversas asignaturas (el proyecto lo estamos implementando en diversas asignaturas, $\mathrm{y}$ tras su utilización ya hemos enviado diversos cuestionarios a los estudiantes preguntándoles sobre las características de la web y su incidencia en su desempeño educativo, su motivación...). Para ello estamos siguiendo sugerencias de autores como Buettner (2013), Maguth et al (2010), Garrigós et al (2015), o Junco et al (2011)

A su vez, estamos utilizando la herramienta aplicando varios criterios de "gamification", incentivando a los alumnos a observar nuevas informaciones que incorporamos como noticias de prensa, videos, etc., relacionados con los contenidos docentes. Estas informaciones o contenido diverso se les han suministrado a los alumnos, y se les ha pedido comentarios sobre las mismas. Los mejores comentarios se han gratificado con puntuaciones extra. Los resultados de estos aspectos todavía están pendientes de analizar.

No obstante, en el desarrollo metodológico del uso de twitter en el aula, hemos detectado también algunos de los inconvenientes de su uso mencionados en la literatura. Aunque no es propósito de este artículo profundizar en el análisis de las desventajas de Twitter (esto lo dejamos para posteriores trabajos), debemos remarcar inconvenientes observados como el riesgo a la privacidad, señalada por algunos alumnos; el sobre trabajo que puede representar para los estudiantes (aspecto también señalado por Garrigós et al (2015) cuando analizaron la utilización de otras redes sociales como Facebook); su desconocimiento por parte de diversos estudiantes (y reticencia también a su utilización); o su limitado uso comparado con otras redes sociales (Johri et al., 2014). Por ejemplo, algunos estudiantes nos mencionaron su preferencia por redes sociales más visuales, cuestión también observada al analizar la evolución y desarrollo de Twitter, frente a otras redes sociales con una creciente relevancia, tales como Youtube o Instagram.

\section{Conclusiones}

El uso de las redes sociales a nivel universitario, es un tópico nuevo, pero con gran desarrollo en los últimos años (Sánchez et al., 2014). Sin embargo, las aplicaciones específicas de los nuevos medios son una cuestión que todavía necesita de investigación teórica y práctica para conocer su incidencia y sus posibilidades.

Como paso previo a su utilización en el aula, este trabajo se ha concentrado en el análisis de las ventajas y posibles usos de Twitter para mejorar la educación a nivel universitario, aunque también ha resaltado escuetamente algunos usos y desventajas de la red. Concretamente, y tras analizar la necesidad de introducir nuevas tecnologías para mejorar la educación, el artículo ha estudiado la relevancia de Twitter, proveyendo una lista extensiva de sus principales ventajas y usos en la educación.

Son muy escasos los estudios que hayan analizado el uso de Twitter en el comportamiento de los estudiantes, aunque existen algunas excepciones que la han utilizado para analizar algunas consecuencias (ejemplo, Dunlap and Lowenthal, 2009; Guzmán et al., 2012; Clark,

(c)) EY-NC-ND 2016, Universitat Politècnica de València

Congreso In-Red (2016) 
2014; Pieterse and Peled, 2014; Thoms and Eryilmaz, 2015). Dado ello, la necesidad de un profundo estudio de este asunto es todavía una carencia importante en la literatura.

Esperamos que este artículo pueda abrir nuevos análisis teóricos y empíricos centrados en los puntos expuestos o en otros posibles desarrollos. El carácter exploratorio del artículo obviamente implica algunas limitaciones, las cuales podrían abrir nuevas líneas de investigación.

En primer lugar, somos conscientes que nuestro estudio es solo el punto de partida para estudiar la relevancia del uso de Twitter en Educación. Aunque en este estudio hemos señalado algunas aplicaciones metodológicas que estamos desarrollando, el análisis empírico del uso de Twitter en educación es todavía escaso. Dado ello, estudios adicionales deberían profundizar en el análisis de ventajas de su utilización con fines educativos. A su vez, podrían crear, por ejemplo a partir de nuestra revisión teórica, escalas y rúbricas sobre el uso de Twitter, y analizar en profundidad las relaciones entre los diferentes usos y los resultados observados por estudiantes. Estas escalas podrían ser validadas con muestras, o por ejemplo con análisis Delphi a expertos, y podrían utilizarse para realizar estudios empíricos. Finalmente, y tal y como hemos comentado, el uso de la "gamificación", y el análisis de su utilización con Twitter también podría abrir nuevas áreas de investigación.

En segundo lugar, el uso de Twitter tiene algunos inconvenientes, que, dado el carácter limitado de este artículo, y aunque algunos se han mencionado, ha sido imposible desarrollar y analizar con profundidad. Estos inconvenientes deberían también ser desarrollados. A su vez, nuestro estudio podría abrir la puerta a un análisis comparado del uso de Twitter frente a otras redes sociales como Facebook o Moodle, que podrían complementar nuestro trabajo.

En tercer lugar, creemos que investigadores y educadores deberían incidir más en el uso de Twitter en el contexto educativo. Consideramos que los educadores deberían desarrollar nuevas aproximaciones y estrategias para ayudar a los estudiantes a utilizar sus comportamientos de redes para mejorar su aprendizaje y desarrollo. El uso de Twitter en clase es crucial para mejorar la educación, dado que los estudiantes de hoy en día, como nativos digitales, están muy familiarizados con el uso de las redes sociales, y ello no puede obviarse. Dado ello, consideramos esencial que este tipo de iniciativas se difundan en el contexto universitario, comenzando por docentes, que se centren fundamentalmente en su uso sobre todo para motivar a los estudiantes (Martínez-Rodrigo, 2013), y a partir de ahí difundir un aprendizaje basado en la colaboración y el trabajo en grupo.

Finalmente, consideramos desacertada en cierta medida la política de las entidades y centros educativos, del desarrollo continuado de aplicaciones informáticas propias, aplicaciones que deben "estudiar" los estudiantes y profesores, siendo de limitado uso sus desarrollo y que incluso obstaculizan el proceso de aprendizaje al detraer tiempo valioso a los alumnos y profesores (el proceso de sobrecarga administrativa a profesores y estudiantes, para el aprendizaje de aplicaciones informáticas diversas, consideramos que es erróneo). Creemos más apropiado la adaptación, uso y promoción de aplicaciones y redes sociales de uso cotidiano, y el esfuerzo de adaptación de las entidades educativas a este uso.

(cc) EY-NC-ND 2016, Universitat Politècnica de València 
En síntesis, creemos que para mejorar la educación deberían ser las entidades educativas las que se adapten a los usos de los estudiantes, más que pedir a estos que aprendan nuevas aplicaciones con uso exclusivo, dado que esto limita la finalidad pretendida por ellas. En consecuencia, consideramos que el estudio y aplicación de las redes sociales de uso cotidiano, y de los comportamientos sociales de los estudiantes, son aspectos necesarios y cruciales para avanzar en el proceso educativo.

\section{Referencias}

-Anikeeva, O., Bywood, P. 2013. "Practice Pointer Social media in primary health care: Opportunities to enhance education, communication and collaboration among professionals in rural and remote locations". Australian Journal of Rural Health 21,132134.

-Barreto, C. R., Jimenez, A. C. 2010. "El uso de Facebook y Twitter en educación”. Lumen-Instituto de Estudios en Educación-IESE 11, 1-9.

-Buettner, R. 2013. "The utilization of Twitter in lectures”. In GI-Jahrestagung (pp. 244254)

-Carpenter, J.P. 2014. "Twitter's capacity to support collaborative learning”. International Journal of Social Media and Interactive Learning Environments 2(2), 103-118.

-Carpenter, J. P., Krutka, D.G. 2014. "How and why educators use Twitter: A survey of the field”. Journal of Research on Technology in Education 46(4), 414-434.

-Castellano, N., Oltra, JV. 2015. "Redes Sociales, conocimiento y comportamiento". ETSINF UPV.

-Clark, M.J. 2014. "Using Twitter to Practice Christian Ethics: Technology and Accompaniment”. Teaching Theology \& Religion 17(3), page 223

-Dunlap, J.C., Lowenthal, P.R. 2009. “Tweeting the night away: Using Twitter to enhance social presence”. Journal of Information Systems Education 20(2), 129-135.

-Evans, C. 2014. "Twitter for teaching: Can social media be used to enhance the process of learning?". British Journal of Educational Technology 45(5), 902-915.

-Falahah, Rosmala, D. 2012. "Study of Social Networking usage in Higher Education Environment”. Procedia - Social and Behavioral Sciences 67 (10), 156-166.

-García-Suárez, J, Trigueros-Cervantes, C., Rivera-García E. 2015. “Twitter como recurso para evaluar el proceso de enseñanza universitaria”. RUSC. Universities and Knowledge Society Journal 12(3), 32-44.

(c)) EY-NC-ND 2016, Universitat Politècnica de València 
-Garrigos-Simon, F. J., Lapiedra Alcamí, R., Barberá Ribera, T. 2012. Social networks and Web 3.0: their impact on the management and marketing of organizations. Management Decision 50(10), 1880-1890.

-Garrigos-Simon, F.J., Oltra, J. V., Montesa-Andres, J. O., Narangajavana, Y., EstellésMiguel, S. 2015. "The use of Facebook and Social Networks to improve Education". Dirección y Organización, 55(April), 4-10.

-Grosseck, G., Holotescu, C. 2008." Can we use twitter for educational activities?’. The 4th International Scientific Conference eLSE "eLearning and Software for Education", Bucharest, April 17-18, 2008. Accessed 15 October, 2015. Retrieved from http://portaldoprofessor.mec.gov.br/storage/materiais/0000012008.pdf

-Guzmán-Duque, A.P., Del Moral-Pérez, M.E., González-Ladrón De Guevara, F. 2012. "Usos de Twitter en las universidades iberoamericanas". Revista Latinoamericana de Tecnología Educativa, 11(1), 27-39.

- Johri, A., Teo, H. J., Lo, J., Dufour, M., Schram, A. 2014. "Millennial engineers: Digital media and information ecology of engineering students". Computers in Human Behavior, 33, 286-301.

-Junco, R., Heiberger, G., Loken, E. 2011. "The effect of Twitter on college student engagement and grades". Journal of Computer Assisted Learning 27(2), 119-132.

-Maduewesi, E. 2013. "Human Resources and Technology: Education in the Days of Google, Wikipedia, Facebook and Twitter". Academic Discourse: An International Journal 5(1), 165-175.

-Maguth, B., Yamaguchi, M., Elliott, J. 2010. "Researching, producing, presenting: Students' use of technology for global advocacy in the social studies". Social Education $74(2), 105-106$.

-Martínez-Rodrigo, E., Raya-González, P. 2014. "El "microblogging" en el proceso de enseñanza-aprendizaje. Una experiencia académica con Twitter". Historia y Comunicación Social 18, 139-147.

-Mendoza, S. 2009. "The Trinity of Community: Google, Facebook and Twitter", Proceedings of World Conference on E-Learning in Corporate, Government, Healthcare, and Higher Education, 2009 (1), 3555-3562.

-Miguel, V., Fernández, M. 2013. "Redes Sociales y Construcción del Conocimiento", in AB Martinez y N. Hernández (Compiadoras),Comunidades Virtuales de Aprendizaje, Caracas, Venezuela. Consejo de Desarrollo Científico y Humanístico, Universidad Central de Venezuela.

(cc) EY-NC-ND 2016, Universitat Politècnica de València Congreso IN-RED (2016) 
-Moody, M. 2010. “Tips for Incorporating Social Media in Traditional Courses". Journal of Magazine \& New Media Research 11(2), 1-9.

-Noguera, I. 2015. "Modelos flexibles de formación: una respuesta a las necesidades actuales". Revista del Congreso Internacional Docencia Universitaria e Innovación. Tarragona.

-Pieterse, E., Peled, Y. 2014. “ A Chaperone: Using Twitter for Professional Guidance, Social Support and Personal Empowerment of Novice Teachers in Online Workshops". Interdisciplinary Journal of E-Learning and Learning Objects 10, 177-194.

-Rinaldo, S.B., Tapp, S., Laverie, D.A. 2011. "Learning by Tweeting: Using Twitter as a pedagogical tool".Journal of Marketing Education, 33(2), 193-203.

-Sammarco, P. 2015. "Estudio sobre los usuarios de Facebook y Twitter en España 2015 The Social Media Family". Accessed 25 October 2015. Retrieved from http://thesocialmediafamily.com/

-Sánchez, R.A., Cortijo, V., Javed, U. 2014. "Students perceptions of Facebook for academic purposes". Computers and Education 70 (1), 138-149

-Stieger, S., Burger, Chr. 2010. "Let as go formative: Continuous student ratings with Web 2.0 application Twitter”. Cyberpsychology, Behavior, and Social Networking 13(2), 163-167.

-Tess, P.A. 2013. "The role of social media in higher education classes (real and virtual)-A literature review”. Computers in Human Behavior 29(5), A60-A68.

-Thoms, B., Eryilmaz, E. 2015. "Introducing a twitter discussion board to support learning in online and blended learning environments". Education and Information Technologies $20(2), 265-283$.

-Twitter. 2016. Accessed 21 January 2016. Retrieved from https://about.twitter.com/company

-Venkatesh, V., Croteau, A. M., Rabah, J. 2014. "Perceptions of effectiveness of instructional uses of technology in higher education in an era of Web 2.0". System Sciences (HICSS), January 2014, 47th Hawaii International Conference on (pp. 110119). IEEE.

-Welch, B. K., Bonnan-White, J. 2012. "Twittering to increase student engagement in the university classroom”. Knowledge Management \& E-Learning: An International Journal (KM\&EL) 4(3), 325-345.

-Westerlund, L., Kaivo-oja, J. 2012. "Digital evolution-from information society to ubiquitous society". Service Design: On the Evolution of Design Expertise, Lahti University of Applied Sciences Series A, Research reports, Part 16, 137-153.

(c)) EY-NC-ND 2016, Universitat Politècnica de València

Congreso In-Red (2016) 
-Wright. N. 2010. "Twittering in teacher education: reflecting on practicum experiences". Open Learning: The Journal of Open and Distance Learning 25(3), 259-265.

-Yang, S.J. (2006). "Context aware ubiquitous learning environments for peer-to-peer collaborative learning”. Journal of Educational Technology \& Society 9(1), 188-201. 